\title{
Research on high-speed synchronous motor direct torque control
}

\author{
Hong Cui1, ${ }^{1,}$, and Youqing $\mathrm{Gao}^{2}$ \\ ${ }^{1}$ Liaoning Provincial College of Communications, Shenyang, Liaoning, China \\ ${ }^{2}$ Shenyang Everbright Environment Technology Co., Ltd., Shenyang, Liaoning, China
}

\begin{abstract}
High-speed motor is increasingly used in ultra-high precision machining and high-performance machinery. It is necessary to achieve closed-loop control of high-speed motor in order to realize highperformance motor control. The high-frequency and high-speed characteristics of high-speed motor have brought great difficulties for its realization of closed-loop control. The performance of the hexagonal flux direct torque control (DTC) method that can reduce the switching frequency of power devices is researched for high-speed synchronous motor in this paper. After adopting this control method, the harmonics in the motor current and torque can be suppressed by adopting an appropriate filtering method. The same effect as the conventional DTC can still be achieved. It provides a basis for the application realization of the highspeed synchronous motor control system.
\end{abstract}

Keywords: Synchronous motor, Hexagonal flux, Closed-loop control, Direct torque control.

\section{Introduction}

Due to the advantages of high speed and high power density as well as its small geometric size, the high-speed motor can effectively save materials. The high-speed motor can be directly connected to the prime mover or load, eliminating the need for conventional mechanical speed change devices. Therefore, the noise can be reduced and the efficiency of the transmission system can be improved, and the dynamic response is faster. Therefore, the research on high-speed motors is currently becoming one of the research hotspots in the field of international electrical engineering ${ }^{[1]}$.

The high-speed synchronous motor has no self-starting ability and the speed adjustment range is wider being high-speed. The starting and debugging is cumbersome easy to lose synchronization. The high-speed motor power supply system has large harmonics. To achieve high-precision control of high-speed motors, closed-loop control must be used. However, due to the high-frequency and high-speed characteristics of high-speed motors, it is difficult to achieve closed-loop control. Seeking a suitable control strategy for high-speed

* Corresponding author: kate_ranran@, 163.com 
motor control systems has become one of the key issues in the control technology of highspeed synchronous motors.

DTC is another high-performance control method of AC motors after vector control. The stator flux orientation is adopted and discrete two-point control is used to make the motor torque respond quickly ${ }^{[2]}$. DTC was initially applied to asynchronous motors. In recent years, although this control method has been initially implemented on synchronous motors, it still needs to be further researched. The inverters of high-speed synchronous motor powered are limited by the switching frequency of power devices, so it is difficult to use the conventional DTC method, and the hexagonal flux square wave DTC method is better. After adopting the hexagonal flux control mode, the 5th and 7th harmonic components in the motor current and torque are relatively large, and suitable filtering methods can be used to suppress them. Based on the mathematical model, simulation is done for the hexagonal flux DTC system of the high-speed synchronous motor in this paper using MATLAB. It is compared with the characteristic simulation of the conventional DTC. The simulation results show that the hexagonal flux DTC method can still achieve the same effect as the conventional DTC, which provides a theoretical basis for the realization of the closed-loop control for the high-speed synchronous motor.

\section{Mathematical model of high speed synchronous motor}

The space vector diagram of the high-speed synchronous motor is shown in Figure 1. In the two rectangular coordinate systems in Figure 1, the $\alpha$ and $\beta$ coordinate systems are the stator stationary coordinate system, and the $\alpha$ axis coincides with the stator winding A axis. The $d$ and $q$ coordinate systems are the rotor rotating coordinate system, and the $d$ axis coincides with the rotor flux direction and rotates counterclockwise at the synchronous speed $\omega$. The angle between the two coordinate systems is $\theta$.

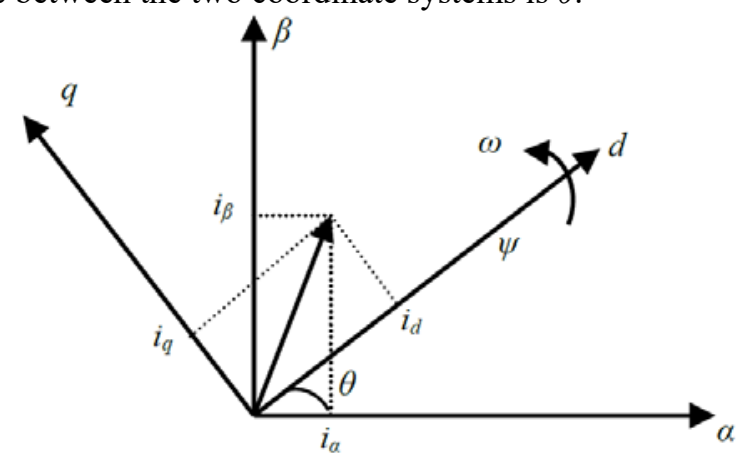

Fig. 1. Diagram of high-speed synchronous motor space vector.

Assuming the magnetic circuit of the high-speed synchronous motor is linear without considering the saturation. The eddy current loss and hysteresis loss of the motor is ignored. The three-phase winding of the motor is completely symmetrical, the motor has no cogging effect, and the air gap magnetic potential of the motor is sinusoidally distributed in space. Then the mathematical model of the high-speed synchronous motor can be obtained as follows:

$$
\frac{\mathrm{d} i_{\mathrm{d}}}{\mathrm{d} t}=\frac{u_{\mathrm{d}}}{L_{\mathrm{d}}}-\frac{R}{L_{\mathrm{d}}} i_{\mathrm{d}}+\frac{L_{\mathrm{q}}}{L_{\mathrm{d}}} i_{\mathrm{q}} p \omega
$$




$$
\begin{gathered}
\frac{\mathrm{d} i_{\mathrm{q}}}{\mathrm{d} t}=\frac{u_{\mathrm{q}}}{L_{\mathrm{q}}}-\frac{R}{L_{\mathrm{q}}} i_{\mathrm{q}}+\frac{L_{\mathrm{d}}}{L_{\mathrm{q}}} i_{\mathrm{d}} p \omega-\frac{\psi_{\mathrm{f}} \omega}{L_{\mathrm{q}}} \\
T=1.5 p_{\mathrm{m}}\left[\psi_{\mathrm{f}} i_{\mathrm{q}}+\left(L_{\mathrm{d}}-L_{\mathrm{q}}\right) i_{\mathrm{d}} i_{\mathrm{q}}\right] \\
\frac{\mathrm{d} \omega}{\mathrm{d} t}=\frac{1}{J}\left(T_{\mathrm{e}}-T_{\mathrm{m}}\right) \\
\frac{\mathrm{d} \theta}{\mathrm{d} t}=\omega
\end{gathered}
$$

In the formula: $u_{\mathrm{d}}$ and $u_{\mathrm{q}}$ are stator voltages of $d$ and $q$ axis; $L_{\mathrm{d}}$ and $L_{\mathrm{q}}$ are the $d$ and $q$ axis self-inductance of equivalent two-phase stator windings; $\Psi_{\mathrm{f}}$ is the flux linkage between permanent magnet poles and stator windings; $T_{\mathrm{e}}$ and $T_{\mathrm{m}}$ are electromagnetic torque and mechanical torque respectively; $p$ is the differential operator; $p_{\mathrm{m}}$ is the number of pole pairs of the torque winding; $J$ is the moment of inertia of the rotor; $\theta$ is the position angle of the rotor; $\omega$ is the mechanical angular velocity of the rotor.

\section{Hexagonal flux DTC of high-speed synchronous motors}

In 1986, Takahashi ${ }^{[3]}$ of Japan and Depenbrock ${ }^{[4]}$ of Germany proposed circular flux trajectory and hexagonal flux trajectory DTC methods for asynchronous motors respectively. With the rapid development of motor technology, many scholars have researched applying the strategy of DTC on synchronous motors, and have achieved certain results. These researches are all for ordinary synchronous motors, and there are few researches on the closed-loop control of high-speed synchronous motors (tens of thousands of revolutions per minute).

The stator torque of the motor is taken as the control object on conventional DTC. The analysis method of space voltage vector is used to directly calculate and control the torque of the motor on the stator coordinate system. According to the two-phase current and voltage of the motor, the flux linkage model and torque model are used to estimate the motor's flux linkage, torque and the sector where the motor stator flux linkage is located in real time. After that, the given torque and flux linkage are respectively compared with the real-time calculated value. Finally, according to the control requirements of the comparison value, the switching vector of the inverter is reasonably selected so that the motor can adjust the output torque according to the control requirements achieving the purpose of speed regulation. Torque and flux linkage are adjusted by two hysteresis regulators respectively. It has the advantages of fast dynamic response, no need for coordinate transformation and insensitivity to motor parameters. It has shortcomings also.

However, it is difficult to adopt the conventional DTC method for the high-speed motor power inverter due to the limitation of the switching frequency of the power device. The hexagonal flux square wave DTC is used in the high-speed synchronous motor control system in this paper. When the flux linkage vector rotates once per revolution, each of the six non-zero voltage vectors is selected only once in turn. Therefore, the requirement for the switching frequency of power devices can be reduced, which is very advantageous for the control of high-speed motors. But the hexagonal flux power supply can only control the power supply frequency, and the control of the power supply current can be achieved by using a chopper to change the amplitude of the voltage vector by adjusting the DC voltage. 
The hexagonal flux square wave DTC sector and space voltage vector selection are shown in Figure 2. The voltage space vector control table is shown in Table 1.

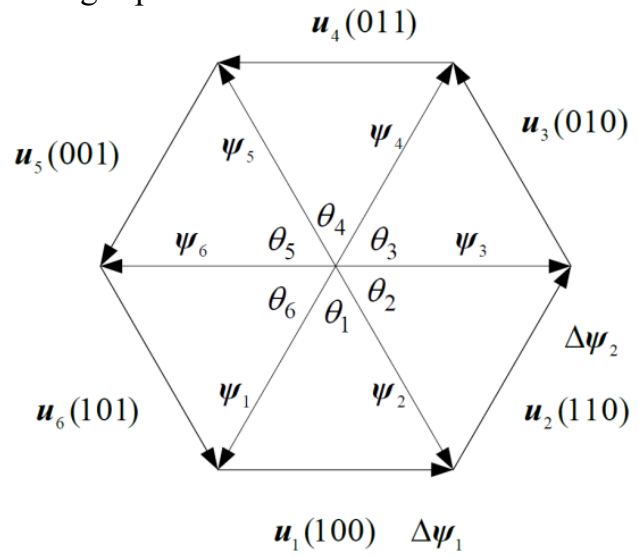

Fig. 2. Hexagonal flux DTC sector and space voltage vector.

Table 1. Hexagonal flux DTC voltage space vector control table.

\begin{tabular}{cccccccc}
\hline$S$ & $T$ & $\theta_{1}$ & $\theta_{2}$ & $\theta_{3}$ & $\theta_{4}$ & $\theta_{5}$ & $\theta_{6}$ \\
\hline 2 & 1 & $u_{1}$ & $u_{2}$ & $u_{3}$ & $u_{4}$ & $u_{5}$ & $u_{6}$ \\
1 & 0 & $u_{4}$ & $u_{5}$ & $u_{6}$ & $u_{1}$ & $u_{2}$ & $u_{3}$ \\
\hline
\end{tabular}

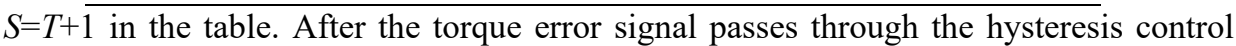
unit of the torque regulator, the 0,1 control signal is obtained, and the voltage space vector is selected according to the above table to realize the rapid adjustment of the torque. Since the flux linkage $\Phi=U / f$, when the inverter output $f$ changes, $U$ changes accordingly controlled by chopper to keep the amplitude of the flux linkage $\Phi$ constant. The relationship between the hexagonal flux DTC flux segment and angle is shown in Table 2.

Table 2. The relationship between hexagonal flux DTC flux segment and angle.

\begin{tabular}{cccc}
\hline angle & segment & angle & segment \\
\hline$[0, \pi / 3)$ & $\theta_{3}$ & {$[\pi / 3,2 \pi / 3)$} & $\theta_{4}$ \\
{$[2 \pi / 3, \pi)$} & $\theta_{5}$ & {$[\pi, 4 \pi / 3)$} & $\theta_{6}$ \\
{$[-2 \pi / 3,-\pi / 3)$} & $\theta_{1}$ & {$[-\pi / 3,0)$} & $\theta_{2}$ \\
\hline
\end{tabular}

After adopting the hexagonal flux DTC method, the 5th and 7th harmonic components in the motor current and torque will increase, and appropriate filtering methods can be used to suppress them. In the research of this paper, an adjustable inductance filter is used. The filter is composed of a low-pass filter and a band-pass filter with adjustable inductance. The band-pass filter can adjust the bandwidth with the change of the output current frequency of the inverter. It is mainly for the 5th and 7th harmonics. The use of adjustable filters can effectively reduce the harmonics in the motor current and torque after the hexagonal flux control method is adopted.

The hexagonal flux DTC simulation model of the high-speed synchronous motor is shown in Figure 3. The model includes stator flux estimation, torque estimation, speed estimation, judgment of flux linkage interval, $3 / 2$ transformation of current and voltage, adjustable inductance filter, chopper and other sub-modules. 


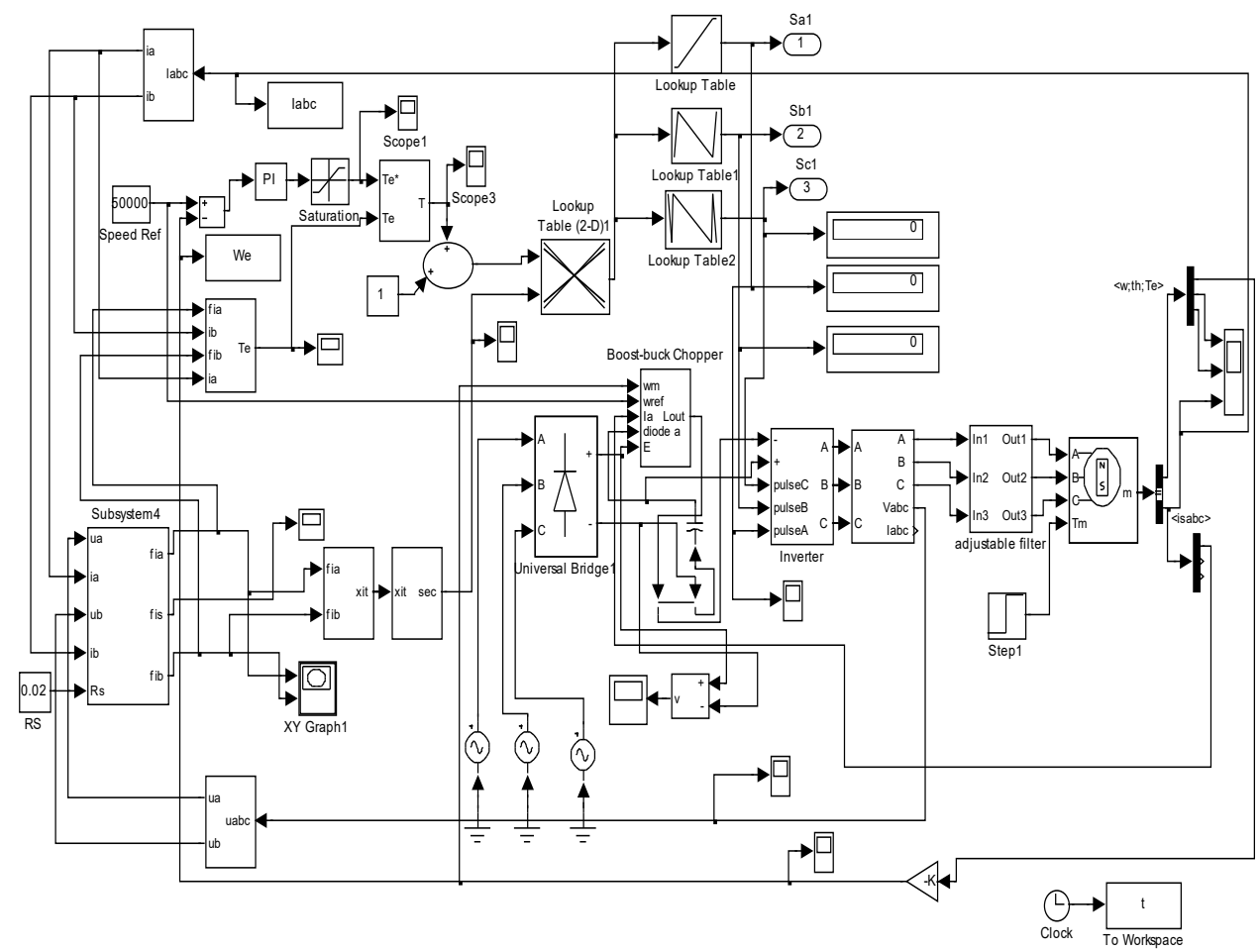

Fig. 3. Hexagonal flux DTC simulation model of high-speed synchronous motor.

\section{System simulation}

In this paper, the controlled object is the high-speed synchronous motor with $P_{\mathrm{e}}=75 \mathrm{KW}$, $U_{\mathrm{e}}=380 \mathrm{~V}, n_{\mathrm{e}}=60000 \mathrm{r} / \mathrm{min}, f_{\mathrm{e}}=1000 \mathrm{~Hz}$, pole pair number $p=1$, stator resistance $R_{\mathrm{s}}=0.02 \Omega$. The hexagonal flux DTC system of the high-speed synchronous motor shown in Figure 3 is simulated. The characteristic simulation is compared with the conventional DTC. The simulation results are shown in Figure 4-Figure 7 which the load of $T=3 \mathrm{~N} . \mathrm{m}$ is suddenly added at $t=0.05 \mathrm{~s}$.

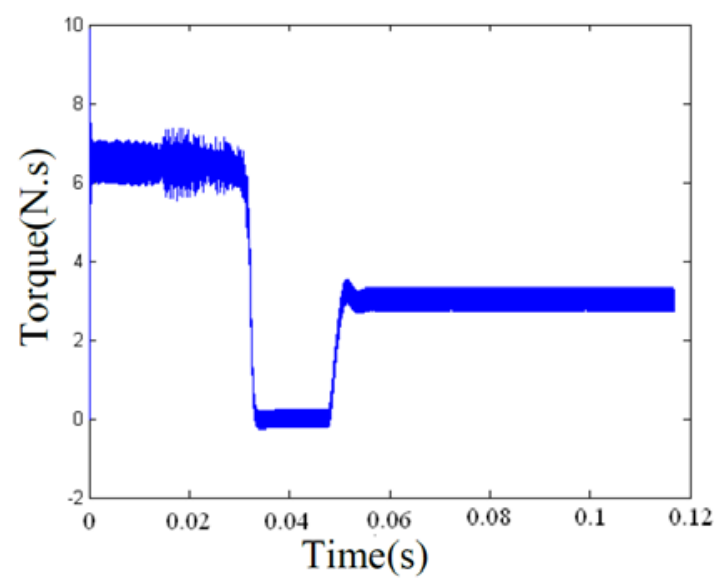

Fig. 4. Torque wave of conventional DTC. 


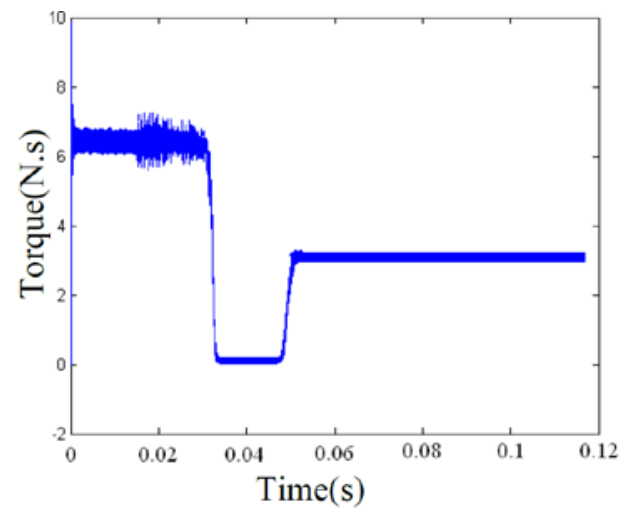

Fig. 5. Torque wave of hexagonal flux DTC with adjustable inductance filter.

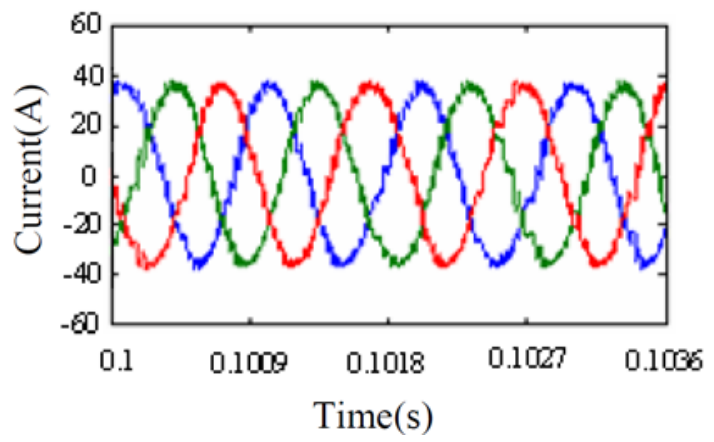

Fig. 6. Stator current wave of conventional DTC.

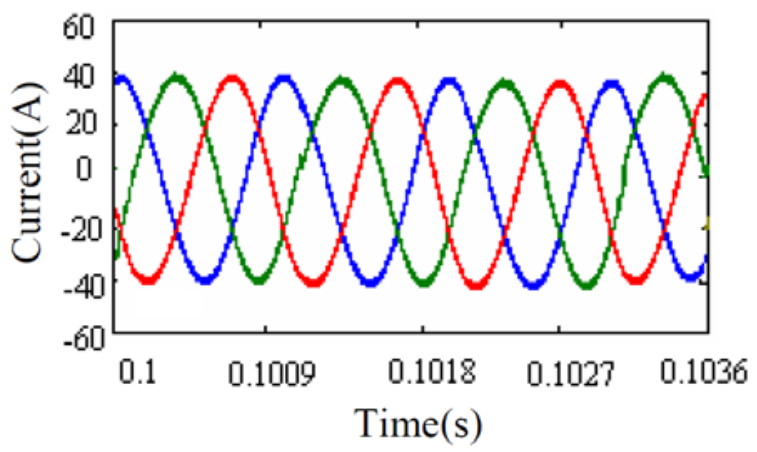

Fig. 7. Stator current wave of hexagonal flux DTC with adjustable inductance filter.

It can be seen from the simulation results that the hexagonal flux DTC method with the appropriate filter can still achieve the same effect as the conventional DTC, which is suitable for the control of high-power high-speed synchronous motor.

\section{Conclusion}

For high-power high-speed synchronous motors, the performance of hexagonal flux DTC that can reduce the switching frequency is researched and a simulation model is established. The hexagonal flux power supply can only control the power supply frequency, and the control of the power supply current can be achieved by using a chopper to change the amplitude of the voltage vector by adjusting the DC voltage. After using the hexagonal flux 
DTC method, the increased 5th and 7th harmonic components in the motor current and torque can be suppressed by using an adjustable inductance filter. It provides a basis for realizing high-precision closed-loop control of high-speed synchronous motors.

\section{References}

1. O. Aglen and A. Anderson. Thermal Analysis of a High-speed Generator. Proc. of IEEE-IAS Annual Meeting, 2003, p 547-554.

2. Han Yingtao, Zhou Qingmiao and Liu Weiguo. Study of Optimal Control Method of REPMSM's VVVF Speed System. Small \& Special Electrical Machines, 2000(2), p 31-33.

3. Takahashi I. and T. Noguchi. A New Quick-response and High-efficiency Control Strategy of an Induction Motor, IEEE Trans. on IA., 1986, 22(5), p 820-827.

4. Depenbrock M. Direct Self-control (DSC) of Inverter Fed Induction Machine. PESC 87 Record: 18th Annual IEEE Power Electronics Specialists Conference (Cat. No.87CH2459-6). IEEE. 1987, p 632-641. 